\title{
THE SUPPORT OF MIKUSIŃSKI OPERATORS
}

\author{
BY
}

THOMAS K. BOEHME $\left({ }^{1}\right),\left({ }^{2}\right)$

ABSTRACT. A class of Mikusiński operators, called regular operators, is studied. The class of regular operators is strictly smaller than the class of all operators, and strictly larger than the class of all distributions with left bounded support. Regular operators have local properties. Lions' theorem of supports holds for regular operators with compact support. The fundamental solution to the CauchyRiemann equations is not regular, but the fundamental solution to the heat equation in two dimensions is regular and has support on a half-ray.

1. Introduction. One of the difficulties in working with Mikusiński operators is that they are defined globally, and except for those operators which can be identified as functions, measures, or distributions, their local properties are not known. There are some Mikusiński operators which are not distributions but whose local properties are rather intuitive. For example the series

$$
a=\sum_{n=0}^{\infty} \frac{s^{n}}{(2 n) !}
$$

is convergent in the field of operators and it seems reasonable that since each of the operators $s^{n}$ is a distribution which is zero on the complement of the origin, the operator $a$ should have zero for its support.

For other operators the local properties are not at all intuitive, for example the fundamental solution for the Laplacian

$$
1 / \Delta=1 /\left(s_{1}^{2}+s_{2}^{2}\right)
$$

or the fundamental solution for the heat operator

$$
1 / \square=1 /\left(s_{1}^{2}-s_{2}\right) \text {. }
$$

In this paper we define a subalgebra of the field of operators which is called the algebra of regular operators. Regular operators have local properties. For example each regular operator has a well-defined support. Every function or distribution with support in a region $R_{a}^{N}=\left\{x \mid x_{i}>\alpha_{i}, i=1, \ldots, N\right\}$ for some $a \in R^{N}$ is a regular operator. There are other regular operators; (1) and (3) above are regular but (2) is not.

Received by the editors February 11, 1972.

AMS (MOS) subject classifications (1970). Primary 44A40, 46F 15, 46F99; Secondary 44A35.

Key words and phrases. Mikusiński operator, distribution, support, finite part, CauchyRiemann operator, heat operator.

(1) This paper was delivered at the International symposium on Mikusiński operators, Dubrovnik, Yugoslavia, June 1971.

(2) This author was supported in part by NSF grant GP-34558. 
A sequence of continuous functions with compact support is said to be an approximate identity if it tends to the $\delta$ function in "nice" manner (Definition 2.6). An operator $a$ is regular if for some approximate identity $\phi_{n}, n=1,2, \cdots$,

$$
a=f_{1} / \phi_{1}=f_{2} / \phi_{2}=\cdots=f_{n} / \phi_{n}=\cdots
$$

where the $f_{n}$ are continuous and have support in some fixed $R_{a}^{N}$.

In $\$ 2$ the Mikusiński operator calculus is developed for $R^{N}$. This is carried out in detail since the notation for the $N$-dimensional calculus is not standardized.

$\$ 3$ contains the explicit statements of what is meant by statements such as "the distribution $T$ is in the field of operators" or "the operator $s$ is the derivative of the $\delta$ function." This is also done in rather great detail since although the terminology has standardized for $N=1$, following Wloka [6], it has not standardized for $N>1$.

$\$ 4$ contains the basic theorems on regular operators. It is shown that the operator

$$
\{(1 / \sqrt{t}) \sin (1 / t)\}^{-1}
$$

and the fundamental solution for the Cauchy-Riemann operator $s_{1}+i s_{2}$ are not regular operators.

In $\$ 5$ we discuss the restriction of operators to open sets.

In $\$ 6$ the support of regular operators is discussed. Lions' theorem on the support of convolution products for distributions with compact support ([3] and [4]) is extended to regular operators with compact support.

The last section contains some examples. The operator in (1) above is shown to be regular and to have the origin for its support. It is shown that $i e^{i \sqrt{s}}$ is a regular operator and that its restriction to $t>0$ is

$$
e^{1 / 4 t / 2 \sqrt{\pi t^{3}}}
$$

It is shown that the fundamental solution for the heat operator in two dimensions ((3) above) is a regular operator and its support is the half-line $x_{1} \geq 0, x_{2}=0$.

Acknowledgement. I would like to thank Professor Melvin Rosenfeld for many helpful discussions while this paper was being propared.

2. Notation. We shall construct the field of Mikusiński operators on $R^{N}$. For any $n$-tuple $a=\left(a_{1}, \cdots, \alpha_{N}\right) \in R^{N}$ let $R_{a}^{N}=\left\{x \mid x \in R^{N}, x=\left(x_{1}, \cdots, x_{N}\right), x_{i} \geq a_{i}\right.$ for each $i=1, \ldots, N\} . C\left(R^{N}\right)$ is the space of continuous functions on $R^{N}$. The support of $f \in C\left(R^{N}\right)$, supp $f$, is the closure of the set on which $f$ is not zero. The space of operators is constructed using the convolution algebra $\mathcal{C}\left(R^{N}\right)=\mathcal{C}$ where

$$
\mathcal{C}\left(R^{N}\right)=\left\{f: f \in C\left(R^{N}\right) \text { and supp } f \in R_{\alpha}^{N} \text { for some } a\right\} .
$$


The convolution of $f$ and $g$ in $\mathcal{C}\left(R^{N}\right)$ is denoted by juxtaposition; thus the function $r=f g$ is given by

$$
\begin{aligned}
r(x)=(f g)(x) & =\int_{-\infty}^{\infty} f(x-t) g(t) d t \\
& =\int_{-\infty}^{\infty} \cdots \int_{-\infty}^{\infty} f\left(x_{1}-t_{1}, \cdots, x_{N}-t_{N}\right) g\left(t_{1}, \cdots, t_{N}\right) d t_{N} \cdots d t_{1}
\end{aligned}
$$

for each $x \in R^{N}$. For each $f \in \mathcal{C}$ the support number of $f, a(f)=\left(a_{1}(f), \ldots, a_{N}(f)\right)$ $\epsilon R^{N}$, is an $n$-tuple with the $i$ th component of $\alpha(f)$ being the number

$$
a_{i}(f)=\operatorname{Sup}\left\{t_{i}: x_{i} \leq t_{i}, x=\left(x_{1}, \ldots, x_{i}, \ldots, x_{N}\right) \Rightarrow f(x)=0\right\} .
$$

Alternatively $\alpha$ is the unique $n$-tuple such that $\operatorname{supp} f \subset R_{a(f)}^{N}$, but if $\beta$ is greater than $\alpha$ in some component then supp $f$ is not contained in $R_{\beta}^{N}$.

The support vector of $f \in \mathcal{C}$ is such that $-\infty<a_{i}(f)<\infty, i=1, \ldots, N$, if $f$ is not the zero function and $\alpha_{i}(0)=\infty$ for $i=1, \ldots, N$. Let [S] denote the convex hull of the set $S$. If $S$ is a closed subset of $R_{\alpha}^{N}$ for some $a$, the convex cap of $S, C(S)$, is defined by

$$
C(S)=\left\{x: \exists x_{0} \in[S] \text { such that }\left(x-x_{0}\right)_{i} \geq 0 \text { for every } i=1, \ldots, N\right\} .
$$

For example if $S$ is the unit circle with center at the origin then

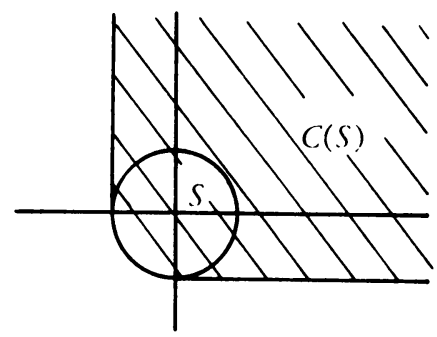

where $C(S)$ is the region.

A theorem of Mikusiński [4] states

Theorem 2.1. If $f$ and $g$ are in $\mathcal{C}$ then $C(\operatorname{supp} f g)=C(\operatorname{supp} f)+C(\operatorname{supp} g)$.

Here the + means the algebraic sum of the two sets in $R^{N}$. Two corollaries are

Corollary 2.2. $\alpha(f g)=\alpha(f)+\alpha(g)$.

Corollary 2.3. $C$ bas no divisors of zero.

The field of operators $\mathbb{N}\left(R^{N}\right)=\mathbb{M}$ is the quotient field of $C$. The support number $a$ can be extended from a homomorphism on the multiplicative semigroup of $\mathcal{C}$ to the reals to become a homomorphism on the multiplicative group of $\pi$ to the reals by the following definition. 
Definition 2.4. Let $a$ be an operator and suppose $a=f / g$ where $f \in \mathcal{C}, g \in \mathcal{C}$, and $g \neq 0$. Then $a(a)=a(f)-a(g)$.

Let $f_{1} / g$ be another representation of the equivalence class $a$. Then $f_{1} / g_{1}=f / g$ implies $f_{1} g=f g_{1}$ and, by Corollary 2.2,

$$
\alpha\left(f_{1}\right)+\alpha(g)=\alpha(f)+\alpha\left(g_{1}\right) \text { or } \cdot a\left(f_{1}\right)-\alpha\left(g_{1}\right)=\alpha(f)-\alpha(g) .
$$

Thus Definition 2.4 is independent of which equivalence class is used to represent $a$.

If $N=1, b$ is the function which is equal to 1 for $x \geq 0$ and $b(x)=0$ for $x<0$. Then $b$ is not in $\mathcal{C}$ but $b$ convolution with itself twice and three times is in $\mathcal{C}$ and $b=b^{3} / b^{2}$ is in $\pi$. The operator $s=b^{-1}$ is the differentiation operator, and $e^{-a_{s}}$ is the translation operator

$$
e^{-\alpha_{s}} f=\{f(t-\alpha)\} \text { for } \alpha \in R^{1} \text {. }
$$

For $\alpha=0$ the translation operator is the identity operator which will be written either as $1,1(x), \delta$, or $\delta(x) ; 1=f / f=\delta$.

Definition 2.5. Let $a \in \mathbb{M}\left(R^{k}\right)$ and $b \in \mathbb{M}\left(R^{l}\right)$. The tensor product $c=a \otimes b \epsilon$ $M\left(R^{k+l}\right)$ is the operator

$$
c=\frac{\left\{f_{1}\left(x_{1}, \cdots, x_{k}\right) f_{2}\left(x_{k+1}, \cdots, x_{k+l}\right)\right\}}{\left\{g_{1}\left(x_{1}, \cdots, x_{k}\right) g_{2}\left(x_{k+1}, \cdots, x_{k+1}\right)\right\}}
$$

where $a=f_{1} / g_{1}$ and $b=f_{2} / g_{2}$.

This definition is again independent of the particular representations $f_{1}, g_{1}$ and $f_{2}, g_{2}$ used for $a$ and $b$.

In particular, we shall use the notations $x_{i}^{\prime}=\left(x_{1}, x_{2}, \ldots, x_{i-1}, x_{i+1}, \ldots, x_{k}\right)$, $b_{i}(x)=b\left(x_{i}\right) \otimes \delta\left(x_{i}^{\prime}\right)$, and $s_{i}(x)=s\left(x_{i}\right) \otimes \delta\left(x_{i}^{\prime}\right)$. Thus for $f \in \mathcal{C}\left(R^{N}\right)$ we have

$$
b_{i} f(x)=\int_{-\infty}^{x_{i}} i\left(x_{i}-t, x_{i}^{\prime}\right) d t
$$

and, for $f$ such that $\partial f / \partial x_{i} \in \mathcal{C}\left(R^{N}\right)$,

$$
s_{i} f(x)=\partial f(x) / \partial x_{i} .
$$

If for example $f$ is a continuously differentiable function in $R_{0}^{N}$ and $f$ is zero outside $R_{0}^{N}$ then

$$
s_{i} f=\{\partial f / \partial x\}+\left\{f\left(0, x_{i}^{\prime}\right)\right\} \otimes \delta\left(x_{i}\right) .
$$

By $S(\epsilon), \epsilon>0$, we mean the $\epsilon$-ball about the origin in $R^{N}$,

$$
S(\epsilon)=\left\{x: x_{1}^{2}+\cdots+x_{N}^{2}<\epsilon^{2}\right\} .
$$


Definition 2.6. By an approximate identity we shall mean a sequence $\phi_{n}$, $n=1,2, \cdots$, such that all the following are satisfied:

(1) $\phi_{n} \in \mathcal{C}\left(R^{N}\right), n=1,2, \cdots$;

(2) for each $\epsilon>0$ there is an $n_{0}(\epsilon)$ such that $n \geq n_{0}$ implies supp $\phi_{n} \subset S(\epsilon)$;

(3) $\phi_{n}(x) \geq 0$ for all $x \in R^{N}$ and for all $n \geq 1$;

(4) $\int_{R}{ }^{N} \phi_{n}(x) d x=1$ for all $n$.

This is an approximate identity in the sense that for any $f \in \mathcal{C}\left(R^{N}\right)$ the convolution products $\phi_{n} f \rightarrow f$ uniformly on the set $S_{a}=\left\{x: x_{i} \leq \alpha_{i}\right\}$ for each $\alpha \in R^{N}$.

Professor Mikusiński calls such a sequence a "delta sequence".

3. Embeddings in $\pi$. There is a natural embedding of $\mathcal{C}\left(R^{N}\right)$ into $\mathbb{M}\left(R^{N}\right)$ given by

$$
g \mid \mathcal{C}\left(R^{N}\right) \rightarrow \mathbb{M}\left(R^{N}\right), \quad g(f)=a_{f} \quad \text { where } a_{f}=f \phi / \phi .
$$

Here $\phi \in \mathcal{C}\left(R^{N}\right)$ is an arbitrary but fixed nonzero function. We will make the identification complete and ordinarily write $f \in \mathbb{M}$.

Besides $\mathcal{C}, \mathbb{M}$ also contains subalgebras which may be identified with algebras of locally integrable functions, Baire measures and distributions.

A. Locally integrable functions. A function $f$ is said to be locally integrable in $R^{N}$ (functions which are equal up to a set of measure zero are identified with each other) if $f$ is integrable on each compact subset of $R^{N}$. For each $\phi \in \mathcal{C}$ which has compact support and each locally integrable $f$,

$$
f * \phi(x)=\int_{R^{N}} f(x-t) \phi(t) d t
$$

is a continuous function in $R^{N}$. If $f \in L_{\text {loc }}^{1}\left(R^{N}\right)$ we will say $f \in \mathbb{M}$ if there exists an $a \in \mathbb{M}$ such that, for each $\phi \in \mathcal{C}\left(R^{N}\right)$ with compact support, $a \phi \in \mathcal{C}\left(R^{N}\right)$ and $f * \phi=a \phi$.

$A$ necessary and sufficient condition that a locally integrable function $f$ be in $M$ is that, for some $a \in R^{N}, f(x)=0$ a.e. in the complement of $R_{a}^{N}$. The convolution algebra of sucb functions is called $\mathcal{L}\left(R^{N}\right)$. For any $\phi \neq 0, \phi \in \mathcal{C}\left(R^{N}\right)$, the mapping $f \rightarrow(f * \phi) / \phi$ is an algebraic isomorphism of $\mathscr{L}\left(R^{N}\right)$ into $\mathbb{M}\left(R^{N}\right)$. The mapping is independent of $\phi$.

In fact the quotient algebra of $\mathcal{Q}$ is the same as that of $\mathcal{C}$. The only thing in the above which we shall prove is the statement that $f \in \mathbb{M}$ implies $f \in \mathcal{L}$. The rest of the statement is very well known.

Let $\phi_{n}$ be an approximate identity and suppose $f \in M$ then $f * \phi_{n}=a \phi_{n} \epsilon$ $\mathcal{C}\left(R^{N}\right)$ and the support vector of $a \phi_{n}$ is

$$
a\left(a \phi_{n}\right)=\alpha(a)+\alpha\left(\phi_{n}\right) \rightarrow a(a)+(0,0, \cdots, 0)=\alpha(a)
$$

as $n$ tends to infinity. Thus for any $\epsilon_{0}>0, a \phi_{n}(x)=0$ outside $R_{\alpha(a)-\epsilon_{0}}^{N}$. But 
since $\phi_{n}$ is an approximate identity for each compact set $K, f * \phi_{n} \rightarrow f$ in $L(K)$ as $n \rightarrow \infty$. Thus on any compact subset of $R^{N}$ disjoint from $R_{\alpha(a)-\epsilon_{0}}^{N}, f$ must equal zero except on a set of measure zero. Since this is true for each $\epsilon_{0}>0$ and for each compact $K, f$ must equal zero almost everywhere outside $R_{a(a)}^{N}$. Thus $f \in \mathfrak{L}\left(R^{N}\right)$.

B. Locally finite measures. If $\mu$ is a locally finite Baire measure on $R^{N}$, then, for each $\phi \in \mathcal{C}\left(R^{N}\right)$ with compact support, $\phi * \mu(x)=\int_{R^{N}} \phi(x-t) d \mu(x)$ is a continuous function on $R^{N}$. We say $\mu=a \in \mathbb{M}$ if, for each $\phi \in \mathcal{C}\left(R^{N}\right)$ with compact support, $\phi * \mu=a \phi$. Again we have

A necessary and sufficient condition that a locally finite Baire measure $\mu$ be in $M$ is that $\mu$ bave support in $R_{\tilde{a}}^{N}$ for some $a \in R^{N}$. The convolution algebra of sucb measures is denoted $\mathfrak{B}\left(R^{N}\right)$. For any nonzero $\phi \in \mathcal{C}\left(R^{N}\right)$ the mapping $\mu \rightarrow$ $(\mu * \phi) / \phi$ is an algebraic isomorpbism of $\mathfrak{B}$ into $\mathbb{N}$ which takes $\mathcal{C}$ onto $\mathcal{C}$ and $£$ onto $\&$.

Again the only part which needs proof is the statement that $\mu \in \mathbb{M} \Rightarrow \operatorname{supp} \mu$ is contained in $R_{\alpha}^{N}$ for some $\alpha$. Since the proof is identical to the proof of the analogous statement for distributions we shall postpone the proof to the discussion of distributions. We shall instead give an example.

Let $\Gamma$ be a smooth rectifiable arc in $R^{N}$ which is contained in $R_{a}^{N}$ for some a. By $b_{\Gamma}$ we mean the measure of arc length on $\Gamma$, i.e., for $f \in \mathcal{C}$,

$$
b_{\Gamma} f(x)=\int_{\Gamma} f(x-t(s)) d s
$$

Example. Suppose $\gamma_{i} \geq 0$ for $i=1, \ldots, N$ and that $\sum_{i=1}^{N} \gamma_{i}^{2}=1$. Let $\Gamma$ be the half-ray

$$
\Gamma=\left\{x \mid x_{i}=\gamma_{i} t, t \geq 0, i=1,2, \cdots, N\right\}
$$

We shall show that

$$
b_{\Gamma}=1 /\left(\gamma_{1} s_{1}+\cdots+\gamma_{N} s_{N}\right) .
$$

Let $H$ be the characteristic function of the set $R_{0}^{N}$; then $H \in \mathscr{Q}, b_{\Gamma} H \in \mathcal{Q}$ and

$$
\begin{aligned}
b_{\Gamma} H(x) & =\int_{\Gamma} H(x-t(s)) d s \\
& =\int_{0}^{\operatorname{Min} 1 \leq i \leq N}\left[x_{i} / \gamma_{i}\right] d s, \quad x \in R_{0}^{N} .
\end{aligned}
$$

Thus

$$
{ }^{b}{ }_{\Gamma}^{H}{ }_{\Gamma}^{H}(x)=\operatorname{Min}_{1 \leq i<N}\left[x_{i} / \gamma_{i}\right], \quad x \in R_{0}^{N},
$$

and 


$$
b_{\Gamma} H(x)=0, \quad x \notin R_{0}^{N} .
$$

Thus for $1 \leq j \leq N, \gamma_{j} s_{j} b_{\Gamma} H(x)=1$ in the set $S_{j}=\left\{x \mid x_{j} / \gamma_{j}=\operatorname{Min}_{1 \leq i \leq N}\left[x_{i} / \gamma_{i}\right]\right\}$ $\cap R_{0}^{N}$ and $\gamma_{j} s_{j} b_{\Gamma} H(x)=0$ in the complement of $s_{j}$. Thus

$$
\sum_{i=1}^{N} \gamma_{i} s_{i} b_{\Gamma} H(x)=H(x)
$$

for all $x$ and thus

$$
b_{\Gamma} \sum_{i=1}^{N} \gamma_{i} s_{i}=1
$$

which is the desired result.

C. Distributions. If $T \in \mathfrak{D}^{\prime}\left(R^{N}\right)$ is a distribution, $\phi$ is infinitely differentiable and has compact support, then $T * \phi$ is an infinitely differentiable function on $R^{N}$. We say $T=a \in \mathbb{M}$, if, for each such $\phi, T * \phi=a \phi . T \in \mathscr{D}^{\prime}\left(R^{N}\right)$ is in $\Pi\left(R^{N}\right)$ if and only if supp $T \subset R_{\alpha}^{N}$ for some a. This set of distributions will be denoted by $\mathscr{D}_{+}^{\prime}\left(R^{N}\right)$. It is a convolution algebra and for any fixed nonzero infinite. ly differentiable $\phi$ in $\mathcal{C}\left(R^{N}\right)$ the mapping $T \rightarrow(T * \phi) / \phi$ is an algebraic isomorphism of $\mathfrak{D}_{+}^{\prime}$ into $\mathbb{M}$ which sends $\mathcal{C}$ onto $\mathcal{C}, \mathfrak{C}$ onto $\mathfrak{Q}$, and $\mathfrak{B}$ onto $\mathfrak{B}$.

Again $\mathscr{D}_{+}^{\prime}\left(R^{N}\right)$ has no divisors of zero and its quotient field is $\mathbb{M}\left(R^{N}\right)$.

We shall prove that if $T \in M\left(R^{N}\right)$ then supp $T \subset R_{\alpha}^{N}$ for some $a$. Let $\phi_{n}$ be an approximate identity which consists of infinitely differentiable functions. If $T=a \in \mathbb{M}$ then $T * \phi_{n}=a \phi_{n}, n=1,2, \cdots$, and support vector of $T * \phi_{n}$ is $\alpha(a)+\alpha\left(\phi_{n}\right)$ which tends to $\alpha(a)$ as. $n \rightarrow \infty$. Thus for each fixed $m, n>m$ implies supp $T * \phi_{n} \subset R_{\alpha(a)}^{N}+S\left(\epsilon_{m}\right)$. Since $T * \phi_{n} \rightarrow T$ in $\mathcal{D}^{\prime}\left(R^{N}\right)$ as $n \rightarrow \infty$, $T$ must be zero in the complement of $R_{a(a)}^{N}+S\left(\epsilon_{m}\right)$ for every $n$. This means $T$ is zero in the complement of $R_{a(a)}^{N}$. Thus supp $T \subset R_{a(a)}^{N}$ which proves the statement.

4. Regular operators. We will say that an operator $a \in \mathbb{M}\left(R^{N}\right)$ is regular if it can be expressed in terms of convolution quotients where the denominator is a "delta sequence" or an approximate identity.

Definition 4.1. An operator $a \in \mathbb{M}\left(R^{N}\right)$ is regular if for each $\epsilon>0$ there exists a $\phi \in \mathcal{C}\left(R^{N}\right)$ with supp $\phi \subset S(\epsilon), \phi(x) \geq 0$ for all $x \in R^{N}$, and $\int_{R^{N}} \phi(x) d x=1$ such that for some $f \in \mathcal{C}\left(R^{N}\right)$ ( $f$ depends on $\phi$ ) we have $a=f / \phi$. Thus there is an approximate identity $\phi_{n}$ such that

$$
a=f_{1} / \phi_{1}=f_{2} / \phi_{2}=\cdots=f_{n} / \phi_{n}=\cdots
$$

Example 4.2. The algebras $\mathcal{C}, \mathcal{L}, \mathfrak{B}$, and $\mathfrak{D}_{+}^{\prime}$ consist of regular operators, 
since, for any infinitely differentiable approximate identity $\phi_{n}$ and any distribution $T \in \mathfrak{D}_{+}^{\prime}\left(R^{N}\right), T=\left(T * \phi_{n}\right) / \phi_{n}$, all $n=1,2, \cdots$.

Theorem 4.3. The regular operators form a subalgebra of M which is properly larger than the algebra $\mathscr{D}_{+}^{\prime}$ and properly smaller than $\mathbb{M}$.

Proof. Let $a$ and $b$ be regular operators and let $a$ and $\beta$ be real numbers. If $a=f_{n} / \phi_{n}$ and $b=g_{n} / \psi_{n}$ we have

$$
\begin{aligned}
\alpha a+\beta b & =\alpha f_{n} / \phi_{n}+\beta g_{n} / \psi_{n}, & & n=1,2, \cdots, \\
& =\alpha f_{n} \psi_{n}+\beta g_{n} \phi_{n} / \phi_{n} \psi_{n}, & & n=1,2, \cdots,
\end{aligned}
$$

and

$$
a b=f_{n} g_{n} / \phi_{n} \psi_{n}, \quad n=1,2, \cdots
$$

Thus it is only necessary to show that if $\phi_{n}$ and $\psi_{n}$ are approximate identities the convolution products $\phi_{n} \psi_{n}$ form an approximate identity.

Properties (1) and (3) of Definition 2.6 are obvious and property (2) follows from Lions' theorem. To verify property (4) we can use the fact that $\int_{R^{N}} \phi(x) d x=1$ if and only if the Fourier transform $\hat{\phi}$ of $\phi$ is 1 at the origin. Since

$$
\widehat{\phi}_{n} \psi_{n}(0)=\hat{\phi}_{n}(0) \hat{\psi}_{n}(0)=1
$$

property (4) is satisfied also.

To show that the algebra of regular operators is strictly between $\mathfrak{D}_{+}^{\prime}$ and $\mathbb{M}$ we will give several examples of operators which are not regular and in $\$ 7$ we give three examples of regular operators which are not in $\mathfrak{D}_{+}^{\prime}\left(R^{N}\right)$.

Example 4.4. Suppose $f(t)=t^{-1 / 2} \sin (1 / t)$ for $t>0$ and $f(t)=0$ for $t<0$. Then $1 / f$ is not regular. It was shown in [2] that this function cannot be made positive by convolution, i.e., there is no nonzero $g$ such that $f g$ is nonnegative. Thus $1 / f \neq g / \phi$ for any nonnegative $\phi$, and $1 / f$ cannot be a regular operator.

Example 4.5. Let $A=s_{1}+i s_{2}$ be the Cauchy-Riemann operator. If $1 / A=$ $f / \phi, \phi, f \in \mathcal{C}\left(R^{2}\right), \phi \neq 0$, we can assume without loss of generality that $f$ and $\phi$ are continuously differentiable; thus

$$
\partial f(x, y) / \partial x+i \partial f(x, y) / \partial y=\phi(x, y)
$$

for all $(x, y) \in R^{2}$. If $\operatorname{supp} \phi \subset S(\epsilon)$, then, in the complement of $S(\epsilon), f$ satisfies the Cauchy-Riemann equation

$$
\partial f / \partial x=-i \partial f / \partial y \quad \text { in } x^{2}+y^{2}>\epsilon^{2}
$$

and is an analytic function of $z=x+i y$. Since $f \in \mathcal{C}\left(R^{2}\right), f$ vanishes when $x$ and $y$ are sufficiently negative and thus $f(z) \equiv 0$ in $\{|z|>\epsilon\}$. Thus the Fourier transform of $\phi$ is 


$$
\hat{\phi}(\xi, \eta)=(i \xi+\eta) \hat{f}(\xi, \eta)
$$

where $\hat{f}$ is the Fourier transform of $f . \hat{\phi}$ and $\hat{f}$ are continuous (in fact, they are entire functions of $\xi$ and $\eta$ ), since they are Fourier transforms of functions with compact support. By $(1), \hat{\phi}(0,0)=0$ and thus

$$
\int_{R} N \phi(x, y) d x d y=\hat{\phi}(0,0)
$$

is zero. It follows that $\phi$ cannot be a positive nontrivial function. In particular $1 / A$ is not a regular operator.

5. Operators on open sets. We shall use the following two well-known properties of approximate identities.

Theorem 5.1. Let $\Omega$ be an open set in $R^{N}$, let $K$ be a compact subset of $\Omega$ and let $f$ be continuous on $\Omega$. Let $\phi_{n}$ be an approximate identity. For suffi:iently large values of $n$ the functions

$$
f \phi_{n}(x)=\int_{R} N(t) \phi_{n}(x-t) d t
$$

are continuous functions on $K$ and the sequence $f \phi_{n}$ converges uniformly to $f$ on $K$ as $n \rightarrow \infty$.

Theorem 5.2. Let $f$ be continuous on $R^{N}$ and suppose supp $\phi \subset S(\epsilon)$; then

$$
\text { supp } f \phi \subset \operatorname{supp} f+S(\epsilon) \text {. }
$$

We will now prove the following theorem.

Theorem 5.3. Let $a \in M, \Omega \subset R^{N}$ open, $f \in C(\Omega)$. The following three statements concerning a are equivalent.

(i) There exists an approximate $\phi_{n}$ such that

$$
a=f_{1} / \phi_{1}=f_{2} / \phi_{2}=\cdots=f_{n} / \phi_{n}=\cdots, \quad f_{n} \in \mathcal{C}\left(R^{N}\right), n=1,2, \cdots,
$$

and the functions $f_{n}$ converge uniformly to $f$ on every compact subset of $\Omega$.

(ii) $a$ is a regular operator and for every approximate identity $\psi_{n}$ such that

$$
a=g_{1} / \psi_{1}=g_{2} / \psi_{2}=\cdots=g_{n} / \psi_{n}=\cdots, \quad g_{n} \in \mathcal{C}\left(R^{N}\right), n=1,2, \cdots,
$$

the sequence $g_{n}$ converges to $f$ uniformly on every compact subset of $\Omega$.

(iii) $a$ is a regular operator with the property that given any compact subset $K$ of $\Omega$ and any $\delta>0$ there is an $\epsilon_{0}=\epsilon_{0}(K, \delta)>0$ such that if $\phi \in \mathcal{C}$, supp $\phi \subset$ $S\left(\epsilon_{0}\right), \int \phi d x=1, \phi(x) \geq 0$ for all $x$ and $a=f_{0} / \phi$, then

$$
\operatorname{Sup}_{K}\left|f_{0}(x)-f(x)\right|<\delta \text {. }
$$


Proof. (ii) and (iii) are clearly equivalent and (ii) trivially implies (i). Thus it is only necessary to show (i) implies (iii). Take $\epsilon_{1}<($ distance $(K, \partial \Omega)) / 2$; then $f$ is uniformly continuous on the neighborhood $K_{0}=K+S\left(\epsilon_{1}\right)$ of $K$. Let $\delta>0$. There is an $\epsilon_{2}<\epsilon_{1}$ such that $x, y \in K_{0}$ and $|x-y|<\epsilon_{2}$ implies $|f(x)-f(y)|<\delta$. Thus

$$
\operatorname{Sup}_{x \in K} \operatorname{Sup}_{|y-x|<\epsilon_{2}}|f(x)-f(y)|<\delta .
$$

Take $\epsilon_{0}(K, \delta)=\epsilon_{2}$ and suppose (i) in the theorem is true. Let $\phi$ be as in (iii) and let $\phi_{n}$ be the approximate identity given in (i). Then $\phi_{n} f_{0}=\phi f_{n}$ and, by Theorem 5.1, $\phi_{n} f_{0}$ tends uniformly on $K_{0}$ to $f_{0}$. Since $f_{n}$ tends uniformly on $K_{0}$ to $f$ and $\phi$ has its support in $S(\epsilon)$ we have, on $K \lim \phi_{n} f_{0}=f_{0}=\lim \phi f_{n}=\phi f$,

$$
f_{0}(x)=\int f(x-t) \phi(t) d t
$$

for every $x \in K$. Thus

$$
f_{0}(x)-f(x)=\int_{S(\epsilon)}[f(x-t)-f(x)] \phi(t) d t
$$

and

$$
\operatorname{Sup}_{x \in K}\left|f_{0}(x)-f(x)\right| \leq \operatorname{Sup}_{x \in K} \operatorname{Sup}_{|x-y|<S(\epsilon)}|f(y)-f(x)| \leq \delta
$$

which proves the theorem.

Definition 5.4. Let $f$ be a continuous function on an open set $\Omega \subset R^{N}$, and let $a$ be a regular operator. We will say that $a(x)$ equals $f(x)$ on $\Omega, a(x)=f(x)$ on $\Omega$, if one of the three equivalent conditions (i), (ii), (iii), of Theorem 5.3 holds.

Theorem 5.5. Let $\Omega_{\alpha}$ be open for each $a \in A$ and suppose that $g_{\alpha} \in C(\Omega)$ for each $a \in A$. Let a be a regular operator sucb that $a(x)=g_{\alpha}(x)$ on $\Omega_{a}$ for each $\alpha \in A$. Then there is a continuous function $g \in C(\Omega), \Omega=\bigcup_{A} \Omega_{\alpha}$ and for each $a \in A, g(x)=g_{a}(x) \quad \forall x \in \Omega_{a}$ and $a(x)=g(x)$ on $\Omega$.

Proof. Let $\phi_{n}$ be an approximate identity and suppose that $a=f_{n} / \phi_{n}$, $n=1,2, \ldots$. Take $\alpha$ and $\beta$ in $A$. We will first show that the restrictions of $g_{a}$ and $g_{\beta}$ to $\Omega_{\alpha \beta}=\Omega_{a} \cap \Omega_{\beta}$ are equal. Let $K_{\alpha \beta}$ be a compact subset of $\Omega_{\alpha \beta}$. Since

$$
a(x)=g_{\alpha}(x) \text { on } \Omega_{\alpha}, \quad a(x)=g_{\beta}(x) \text { on } \Omega_{\beta},
$$

$f_{n}$ converges uniformly on $K_{\alpha \beta}$ to $g_{\alpha}$ and to $g_{\beta}$. Since this is true for every compact subset of $\Omega_{\alpha} \cap \Omega_{\beta}$ we have

$$
\left.g_{a}\right|_{\boldsymbol{\Omega}_{\alpha \beta}}=\left.g_{\beta}\right|_{\boldsymbol{\Omega}_{\alpha \beta}} .
$$

It follows that the $g_{a}$ fit together correctly and there is a $g \in C(\Omega)$ such that 
$\left.g\right|_{\boldsymbol{\Omega}_{\alpha}}=g_{\alpha} \forall \alpha \in A$.

Given a compact $K \subset \Omega$ we have

$$
\begin{array}{ll}
K=\bigcup_{i=1}^{k} K_{i}, & K_{i} \text { compact } \\
K_{i} \subset \Omega_{a_{i}}, & 1 \leq i \leq k, \\
\alpha_{i} \in A, & 1 \leq i \leq k,
\end{array}
$$

and since the sequence $f_{n}$ converges uniformly on each $K_{i}, 1 \leq i \leq n$, it follows that $f_{n}$ converges uniformly on each compact subset $K$ of $\Omega$ to $g$ which completes the proof of the theorem.

Corollary 5.6. If $a$ is a regular operator, $\Omega$ is open, $f \in C(\Omega), g \in C(\Omega)$,

$$
a(x)=f(x) \text { on } \Omega, \text { and } a(x)=g(x) \text { on } \Omega \text {, }
$$

then

$$
f(x)=g(x) \quad \forall x \in \Omega .
$$

Proof. Take $A=\{1,2\}, \Omega_{1}=\Omega=\Omega_{2}, g_{1}=f$ and $g_{2}=g$ in the theorem.

Corollary 5.7. For each regular operator a there is a unique largest open set

(i) on which $a$ is a continuous function;

(ii) on which $a$ is an infinitely differentiable function;

(iii) on which $a$ is equal to zero.

Proof. These are all local properties and thus the corollary follows from the theorem.

Theorem 5.8. Let a be regular and $f \in C\left(R^{N}\right)$; then $a(x)=f(x)$ on $R^{N}$ if and only if $f \in \mathcal{C}\left(R^{N}\right)$ and $a=f$.

Proof. The if part of the theorem follows from Theorem 5.1. We will prove the only if part. If $a=f_{n} / \phi_{n}$ for an approximate identity $\phi_{n}$, then $\phi_{n} a \rightarrow a$ in $\mathcal{C}\left(R^{N}\right)$ since the $\phi_{n} a$ converge uniformly on compact sets in $R^{N}$ and the support vectors $a\left(\phi_{n} a\right)=\alpha\left(\phi_{n}\right)+\alpha(a) \rightarrow a(a)$ are bounded below which completes the proof.

Theorem 5.9. If $a$ and $b$ are regular and $\Omega \subseteq R^{N}$ is open, then $a(x)=f(x)$ on $\Omega, b(x)=g(x)$ on $\Omega$ implies $(a+b)(x)=f(x)+g(x)$ on $\Omega$.

The proof is similar to the proof of Theorem 5.3.

6. The support of regular operators. We make the following definition of the support of regular operators. 
Definition 6.1. If $a$ is a regular operator then the support of $a, \operatorname{supp} a$, is the complement of the largest open set on which $a$ is zero.

Theorem 6.2. An operator bas compact support if and only if there is an ap. proximate identity such that, for every $n=1,2, \cdots, a=f_{n} / \phi_{n}$ and the functions $f_{n}$ all bave support in some fixed compact set $K$.

Proof. Certainly such an operator vanishes outside $K$ and thus $a$ has compact support.

Conversely, suppose $a$ has compact support $K$. If $\phi_{n}$ is an approximate identity such that

$$
a=f_{n} / \phi_{n}=f_{m} / \phi_{m}, \quad n=1,2, \cdots, m=1,2, \cdots,
$$

we have

$$
\phi_{m} f_{n}=\phi_{n} f_{m}, \quad n=1,2, \cdots, m=1,2, \cdots,
$$

and if $\phi_{n}$ has support in $S\left(\epsilon_{n}\right)$, then in the complement of $K+S\left(\epsilon_{n}\right)$ we have

$$
f_{n}(x)=\lim _{m \rightarrow \infty} \phi_{m} f_{n}(x)=\lim _{m \rightarrow \infty} \phi_{n} f_{m}(x)=0 .
$$

Thus supp $f_{n} \subset K+S\left(\epsilon_{n}\right)$ which proves the theorem.

In fact we have shown that

$$
\operatorname{supp} a+S\left(\epsilon_{n}\right) \supset \operatorname{supp} f_{n}
$$

for each $\phi_{n}$ and $f_{n}, n=1,2, \ldots$. Conversely if $a$ has compact support and $\epsilon>0$ is any fixed positive number there is an $n_{0}=n_{0}(\epsilon)$ such that for all $n>n_{0}(\epsilon)$

$$
\operatorname{supp} f_{n}+S(\epsilon) \supset \operatorname{supp} a
$$

since, for any fixed point $x_{0} \in \operatorname{supp} a$, there is an $n\left(x_{0}\right)$ such that $n>n\left(x_{0}\right)$ implies supp $f_{n}+S(\epsilon)$ contains $x_{0}$. The compactness of supp $a$ then implies that there is a single $n_{0}=n_{0}(\epsilon)$ such that (3) holds for $n>n_{0}$.

We can now extend the theorem of Lions on compact supports to regular operators.

Theorem 6.3. If $a$ and $b$ are regular operators with compact support then $a b$ bas compact support and

$$
[\operatorname{supp} a b]=[\operatorname{supp} a]+[\operatorname{supp} b]
$$

where [ ] denotes the convex bull and + denotes the vector sum of the two sets.

Proof. Let $a$ be an operator with compact support. Suppose that $\phi_{n}$ is an approximate identity and $a=f_{n} / \phi_{n}$ with supp $\phi_{n} \subset S\left(\epsilon_{n}\right)$ then taking convex hulls in (2) we have 


$$
[\operatorname{supp} a]+S\left(\epsilon_{n}\right) \supset\left[\operatorname{supp} f_{n}\right]
$$

and taking convex hulls in (3) gives

$$
\left[\operatorname{supp} f_{n}\right]+S(\epsilon) \supset[\operatorname{supp} a]
$$

if $n$ is sufficiently large.

Let $a=f_{n} / \phi_{n}$ and $b=g_{n} / \psi_{n}$ where $\phi_{n}$ and $\psi_{n}$ are approximate identities. By $\left(3^{\prime}\right)$ we have

$$
\begin{aligned}
{[\operatorname{supp} a b] } & \subset\left[\operatorname{supp} f_{n} g_{n}\right]+S(\epsilon) \\
& \subset\left[\operatorname{supp} f_{n}\right]+\left[\operatorname{supp} g_{n}\right]+S(\epsilon) \text { by Lions' theorem, } \\
& \subset[\operatorname{supp} a]+[\operatorname{supp} b]+S(\epsilon)+2 S\left(\epsilon_{n}\right) \text { by }\left(2^{\prime}\right)
\end{aligned}
$$

for every $\epsilon>0$ when $n$ is sufficiently large. Taking the intersection for all $\epsilon>0$ gives

$$
[\operatorname{supp} a b] \subset[\operatorname{supp} a]+[\operatorname{supp} b]
$$

A similar application of first $\left(2^{\prime}\right)$, then Lions's theorem, then $\left(3^{\prime}\right)$ and finally letting $\epsilon$ tend to zero yields

$$
[\operatorname{supp} a]+[\operatorname{supp} b] \subset[\operatorname{supp} a b] .
$$

Inclusions (2") and (3") yield Theorem 6.3.

7. Examples. Since every distribution with support in $R_{\alpha}^{N}$ for some $\alpha$ is a regular operator we shall only give examples of operators which are not distributions.

Example 7.1. Let $a$ be the operator

$$
a=\sum_{k=0}^{\infty} \frac{s^{k}}{(2 k) !} .
$$

Since the sequence $(2 k)$ ! defines a class of infinitely differentiable functions which is not quasi-analytic in the sense of Denjoy [5, Chapter $19, \$ 19.5]$ there is an approximate identity $\phi_{n}$ such that, for each $n$,

(i) $\phi_{n}$ is infinitely differentiable;

(ii) $\operatorname{supp} \phi_{n} \subset S(1 / n)$;

(iii) $\sum_{k=1}^{\infty} \phi_{n}^{(k)} /(2 k)$ ! is uniformly convergent on $R^{1}$.

Thus $a$ is a regular operator

$$
a=\left\{\sum_{k=1}^{\infty} \frac{\phi_{n}^{(k)}(t)}{(2 k) !}\right\} / \phi_{n}, \quad n=1,2, \ldots,
$$

and, for each $n, a \phi_{n}$ vanishes outside $S(1 / n)$. Thus supp $a=\{0\}$. 
Before going to the next example we will make some observations on the convergence of regular operators.

Definition 7.2. Let $a_{k}, k=1,2, \cdots$, be a sequence of regular operators. We will say that $a_{k}$ tends to $a(\pi R)$ as $k \rightarrow \infty$ if there is an approximate identity $\phi_{n}, n=1,2, \cdots$, such that

$$
a_{k}=f_{n, k} / \phi_{n}, \quad f_{n, k} \in \mathcal{C}, n=1,2, \cdots, k=1,2, \cdots,
$$

and for each fixed $n$ the sequence $f_{n, k}$ converges in $\mathcal{C}$ to an $f_{n}$ where $a=f_{n} / \phi_{n}$, $n=1,2, \cdots$ (a sequence $g_{k} \in \mathcal{C}\left(R^{N}\right)$ converges in $\mathcal{C}$ if there is a $a \in R^{N}$ such that $a\left(g_{k}\right) \geq \alpha$ for all $k$ and $g_{k}$ converges uniformly on compact sets to $g$ ).

If $a_{k} \rightarrow a(\pi R)$ then $a$ is also regular. The following theorem on regular convergence of operators is an easy consequence of the definition.

Theorem 7.3. Let $a_{k}$ be regular operators whicb converge to a $(\Re R)$ as $k \rightarrow \infty$. Suppose that for some fixed open set $\Omega$ there are functions $g_{k} \in C(\Omega)$ such that $a_{k}(x)=g_{k}(x)$ on $\Omega$. If the sequence $g_{k}$ converges uniformly on compact subsets of $\Omega$ to $g$ then $a(x)=g(x)$ on $\Omega$.

In [1] the operator $i e^{i \sqrt{s}}$ is used as a finite part.

$$
F P e^{1 / 4 t} / 2 \sqrt{\pi t^{3}}=i e^{i \sqrt{s}} \text {. }
$$

Example 7.4. We will show that the operator $i e^{i \sqrt{s}}$ is regular, that its singular support (the complement of the set on which it is an infinitely differentiable function) is the origin, and that

$$
i e^{i \sqrt{s}}(x)=e^{1 / 4 x} / 2 \sqrt{\pi x^{3}} \text { on } x>0 .
$$

The series expansions

$$
i e^{i \sqrt{s}}=\sum_{n=0}^{\infty} \frac{(i)^{n+1} s^{n / 2}}{n !}, \quad a_{1}=i \sum_{k=0}^{\infty} \frac{(-1)^{k} s^{k}}{(2 k) !}, \quad a_{2}=\sum_{k=0}^{\infty} \frac{(-1)^{k+1} s^{k+1 / 2}}{(2 k+1) !}
$$

are convergent in $\mathbb{M}$ and $i e^{i \sqrt{s}}=a_{1}+a_{2}$. The operator $a_{1}$ is essentially the operator which appears in Example 7.1 and the method used there shows that $a_{1}$ has $\{0\}$ for its support. However, we will use Theorem 7.3 here to determine the restriction of $a_{1}$ and $a_{2}$ to $\Omega=\{x: x \neq 0\}$.

We will use the fact that, for each $k, k=0,1,2, \cdots$,

$$
\begin{aligned}
s^{k}(x) & =0 \quad \text { for } x \neq 0, \\
s^{k+1 / 2}(x) & =1 / x^{(k+3 / 2)} \Gamma(-(k+1 / 2)) \text { for } x>0, \\
s^{k+1 / 2}(x) & =0 \text { for } x<0 .
\end{aligned}
$$

(These results can be proved directly from the definition; they are proved in [1, 
p. 359, Table of Finite Parts, entry 7, and Theorem 7], since the operators involved are distributions.)

It follows immediately from Theorem 7.3 that

$$
\begin{aligned}
a_{1}(x) & =0 \text { for } x \neq 0, \\
a_{2}(x) & =0 \text { for } x<0 \\
a_{2}(x) & =\sum_{k=0}^{\infty} \frac{(-1)^{k+1}}{(2 k+1) !} \frac{1}{x^{k+3 / 2} \Gamma(-(k+1 / 2))} \text { for } x>0, \\
& =\frac{1}{\Gamma(1 / 2) 2 x^{3 / 2}} \sum_{k=0}^{\infty} \frac{1}{k !} \frac{1}{(4 x)^{k}} \quad \text { for } x>0, \\
a_{2}(x) & =\frac{e^{1 / 4 x}}{2 \sqrt{\pi x^{3}}} \text { for } x>0 .
\end{aligned}
$$

Thus the support of $i e^{i \sqrt{s}}$ is the half-line $x \geq 0$, and the restriction to $x>0$ is

$$
i e^{i \sqrt{s}}(x)=e^{1 / 4 x} / 2 \sqrt{\pi x^{3}}, x>0 .
$$

Example 7.5. Let $a=\left(s_{1}^{2}-s_{2}\right)^{-1}$ be the fundamental solution to the heat equation in $R^{2}$. The support of $a$ is the half-line

$$
\underbrace{}_{\operatorname{supp} a} a=0 \quad x_{1} \geq 0, x_{2}=0 .
$$

Since $a=b_{1}^{2} /\left(1-s_{2} b_{1}^{2}\right)=b_{1}^{2} \Sigma_{k=0}^{\infty} s_{2}^{k} b_{1}^{2 k}$, we will take an approximate identity $\phi_{n}\left(x_{1}, x_{2}\right)=\psi_{n}\left(x_{1}\right) \psi_{n}\left(x_{2}\right)$ where $\psi_{n}$ is an approximate identity in one variable. By the Denjoy-Carlemann theorem $\left[5\right.$, p. 376] we can pick $\psi_{n}$ such that
(1) $\psi_{n}$ has support in $(-1 / n, 1 / n)$,
(2) $\psi_{n}$ is infinitely differentiable,
(3) For each $n,\left[\left(\operatorname{Max}\left|\psi_{n}^{(k)}(x)\right|\right) /(2 k)\right]^{1 / k} \rightarrow 0$ as $k \rightarrow \infty$.

Then

$$
a \phi_{n}=\sum_{n=0}^{\infty} \psi_{n}^{(k)} \otimes b^{2 k+2} \psi_{n}
$$

is uniformly convergent on $R^{2}$ to a continuous function with support in 
$\left\{x\left|x_{1}>1 / n,\right| x_{2} \mid<1 / n\right\}$. Thus

$$
\text { supp } a \subset\left\{x \mid x_{1} \geq 0, x_{2}=0\right\} \text {. }
$$

Since given any neighborhood of a point on the positive $x_{1}$-axis there is a sufficiently large $n$ such that $a \phi_{n}$ fails to vanish in that neighborhood we have

$$
\operatorname{supp} a=\left\{x \mid x_{1} \geq 0, x_{2}=0\right\} \text {. }
$$

Added in proof. In On power series in the differentiation operator, Studia Math. (to appear), it is shown that every power series $x=\Sigma A_{n} s^{n}$ which converges in the usual convergence in Mikusiński operators represents a regular operator and supp $a=\{0\}$.

\section{REFERENCES}

1. T. K. Boehme, Operational calculus and the finite part of divergent integrals, Trans. Amer. Math. Soc. 106 (1963), 346-368. MR $26 \# 1712$.

2. - Convergence of positive functions in the space of Mikusinski operators, Proc. Amer. Math. Soc. 17 (1966), 12-17. MR $32 \# 4477$.

3. J. L. Lions, Supports dans la transformation de Laplace, J. Analyse Math. 2 (1953), 369-380. MR 15, 307.

4. J. G. Mikusiński, Convolution of functions of several variables, Studia Math. 20 (1961), 301-312. MR 25\#4309.

5. W. Rudin, Real and complex analysis, McGraw-Hill, New York, 1966. MR 35 \#1420.

6. J. Wloka, Distributionen und Operatoren, Math. Ann. 140 (1960), 227-244. MR 22 \#4946.

DEPARTMENT OF MATHEMATICS, UNIVERSITY OF CALIFORNIA, SANTA BARBARA, CALIFORNIA 93106 